\title{
OCT study of skin optical clearing with preliminary laser ablation of epidermis
}

\author{
Natalia S. Ksenofontova ${ }^{1 *}$, Elina A. Genina ${ }^{1,2}$, Alexey N. Baskatov ${ }^{1,2}$, Georgy S. Terentyuk ${ }^{3}$, \\ and Valery V. Tuchin ${ }^{1,4,5}$ \\ ${ }^{1}$ Saratov National Research State University, 83 Astrakhanskaya Str., Saratov 410012, Russia \\ ${ }^{2}$ National Research Tomsk State University, 36 Lenina Ave., Tomsk 634050, Russia \\ ${ }^{3}$ The First Veterinary Clinic, 98 Astrakhanskaya Str., Saratov 410012, Russia \\ ${ }^{4}$ Institute of Precision Mechanics and Control, Russian Academy of Sciences, Laboratory of Laser Diagnostics of \\ Technical and Living Systems, 24 Rabochaya Str., Saratov 410028, Russia \\ ${ }^{5}$ Samara National Research University, 34 Moskovskoe Shosse, Samara 443086, Russia \\ * e-mail: Natasha13.95@mail.ru
}

\begin{abstract}
We report the results of the experimental study of optical immersion clearing of laboratory animals skin in vivo with preliminary laser ablation of epidermis. It is shown that the ablation of the skin surface leads to the local edema that reduces the optical detection depth immediately after the impact. However, the water evaporation from the damaged epidermis causes the optical clearing of skin, comparable with that caused by polyethylene glycol in intact skin. It is shown that the preliminary ablation of the skin surface before the application of immersion agent does not lead to significant increase of the optical detection depth as compared to the sole effect of ablation or immersion. (C) 2017 Journal of Biomedical Photonics \& Engineering.
\end{abstract}

Keywords: skin laser ablation; optical coherence tomography; optical detection depth; immersion optical clearing.

Paper \#3141 received 3 Mar 2017; revised manuscript received 5 Apr 2017; accepted for publication 5 Apr 2017; published online 27 Apr 2017. doi: 10.18287/JBPE17.03.020307. [Special Issue. Workshop "Biophotonics" of the XIII all-Russian Youth Samara conference-contest on optics and laser physics].

\section{References}

1. V. V. Tuchin, "Tissue optics and photonics: biological tissue structures," Journal of Biomedical Photonics \& Engineering 1(1), 3-21 (2015).

2. V. V. Tuchin, "Tissue optics and photonics: light-tissue interaction," Journal of Biomedical Photonics \& Engineering 1(2), 98-134 (2015).

3. D. Zhu, K. Larin, Q. Luo, and V. V. Tuchin, "Recent progress in tissue optical clearing," Laser \& Photonics Reviews 7(5), 732-757 (2013).

4. E. A. Genina, A. N. Bashkatov, Yu. P. Sinichkin, I. Yu. Yanina, and V. V. Tuchin, "Optical clearing of biological tissues: prospects of application in medical diagnostics and phototherapy," Journal of Biomedical Photonics \& Engineering 1(1), 22-58 (2015).

5. S. Karma, J. Homan, C. Stoianovici, and B. Choi, "Enhanced fluorescence imaging with DMSO-mediated optical clearing," Journal of Innovative Optical Health Sciences 3(3), 153-158 (2010).

6. H. Zhong, Z. Guo, H. Wei, L. Guo, C. Wang, Y. He, H. Xiong, and S. Liu, "Synergistic effect of ultrasound and Thiazone PEG 400 on human skin optical clearing in vivo," Photochem. Photobiol. 86(3), 732-737 (2010).

7. J. Yoon, D. Park, T. Son, J. Seo, J. S. Nelson, and B. Jung, "A physical method to enhance transdermal delivery of a tissue optical clearing agent: Combination of microneedling and sonophoresis," Lasers Surg. Med. 42(5), 412-417 (2010).

8. X. Xu, and Q. Zhu, "Feasibility of sonophoretic delivery for effective skin optical clearing," IEEE Trans. Biomed. Eng. 55(4), 1432-1437 (2008). 
9. H. Zhong, Z. Guo, H. Wei, C. Zeng, H. Xiong, Y. He, and S. Liu, "In vitro study of ultrasound and differentconcentration glycerol-induced changes in human skin optical attenuation assessed with optical coherence tomography," J. Biomed. Opt. 15(3), 036012 (2010).

10. C.-H. Lin, I. A. Aljuffali, and J.-Y. Fang, "Lasers as an approach for promoting drug delivery via skin," Expert Opinion on Drug Delivery 11(4), 599-614 (2014).

11. I. A. Aljuffali, C. F. Lin, and J. Y. Fang, "Skin ablation by physical techniques for enhancing dermal/transdermal drug delivery," J. Drug Del. Sci. Tech. 24(3), 277-287 (2014).

12. X. Xu and Q. Zhu, "Sonophoretic delivery for contrast and depth improvement in skin optical coherence tomography," IEEE Journal of Selected Topics in Quantum Electronics 14(1), 56-61 (2008).

13. X. Wen, S. L. Jacques, V. V. Tuchin, and D. Zhu, "Enhanced optical clearing of skin in vivo and optical coherence tomography in-depth imaging," J. Biomed. Opt. 17(6), 066022 (2012).

14. K. V. Larin, M. G. Ghosn, A. N. Bashkatov, E. A. Genina, N. A. Trunina, and V. V. Tuchin, "Optical clearing for OCT image enhancement and in-depth monitoring of molecular diffusion," IEEE Journal of Selected Topics in Quantum Electronics 18(3), 1244-1259 (2012).

15. E. A. Genina, A. N. Bashkatov, A. A. Korobko, E. A. Zubkova, V. V. Tuchin, I. V. Yaroslavsky, and G. B. Altshuler, "Optical clearing of human skin: comparative study of permeability and dehydration of intact and photothermally perforated skin,” J. Biomed. Opt. 13(2), 021102 (2008).

16. J. S. Nelson, J. L. McCullough, T. C. Glenn, W. H. Wright, L.-H. L. Liaw, and S. L. Jacques, "Mid-infrared laser ablation of stratum corneum enhances in vitro percutaneous transport of drugs," J. Invest. Dermatol. 97(5), 874-879 (1991).

17. A. Fujiwara, T. Hinokitani, K. Goto, and T. Arai, "Partial ablation of porcine stratum corneum by argonfluoride excimer laser to enhance transdermal drug permeability," Lasers Med. Sci. 19, 210-217 (2005).

18. H.-J. Jang, E. Hur, Y. Kim, S.-H. Lee, N. G. Kang, and J. J. Yoh, "Laser-induced microjet injection into preablated skin for more effective transdermal drug delivery," J. Biomed. Opt. 19(11), 118002 (2014).

19. E. A. Genina, A. N. Bashkatov, L. E. Dolotov, G. N. Maslyakova, V. I. Kochubey, I. V. Yaroslavsky, G. B. Altshuler, and V. V. Tuchin, "Transcutaneous delivery of micro- and nanoparticles with laser microporation," J. Biomed. Opt. 18(11), 111406 (2013).

\section{Introduction}

Skin has a complex inhomogeneous structure causing a strong light scattering, which limits the optical methods applicability to the diagnostics and treatment of various diseases $[1,2]$. The development of methods enhancing the optical clearing of skin is an urgent problem that has been a subject of multiple studies [3, 4]. However, the diffusion of immersion agents into the skin is hindered by the existence of epidermal barrier. To overcome the barrier function of the epidermis, different methods are used, including the chemical dissolving of the lipid layer [5, 6], the mechanical damages [7], the effect of ultrasound [6-9], etc. Among these methods, the laser microablation of epidermis can be used to solve the problem. In the literature, the results of applying microablation to enhance the skin permeability for different medical preparations and particles are presented [10, 11]. However, the effect of the epidermis microablation on the optical detection depth in the case of monitoring the process of skin optical clearing using the optical coherence tomography (OCT) is studied insufficiently.

OCT is widely used to study the skin optical clearing, since it is a relatively simple and convenient instrument for monitoring the changes of optical probing depth and attenuation coefficient in the process of the immersion agent penetration into the skin $[9$, 12-14].

The aim of the present work is to study the effect of the epidermis ablation in vivo on the optical detection depth using OCT in the process of skin immersion optical clearing. The paper presents the results of the comparative study of the immersion clearing of the intact skin and the skin with preliminary ablation of the surface epidermis layer.

\section{Methods and Materials}

As a source of radiation for skin ablation we used the erbium laser Palomar Lux2940 (Palomar Medical Technologies Inc., Burlington, MA, USA) generating single-spike pulses at the wavelength $2940 \mathrm{~nm}$ with the energy of $1 \mathrm{~J}$, the pulse duration being $5 \mathrm{~ms}$, and the spike duration being $200 \mu \mathrm{s}$. In the experiments, we used the regime of ablation of the upper layer of skin using a wide beam at the area $6 \times 6 \mathrm{~mm}^{2}$ to the depth not exceeding $50 \mu \mathrm{m}$. As an illustration, Fig. 1 shows a fragment of skin with the epidermis ablation.

As an optical clearing agent, we used polyethylene glycol 300 (PEG-300, the molecular weight 300 dalton, Sigma-Aldrich, Germany). The refractive index of PEG300 at the wavelength $930 \mathrm{~nm}$ is equal to 1.456 . The measurements were carried out using the multiwave Abbe refractometer DR-M2/1550 (ATAGO, Japan).

The studies were performed in vivo in 15 laboratory albino rats, divided into three groups of 5 animals in each: group I - laser ablation alone, group II application of PEG-300 alone, and group III - ablation followed by PEG application. The age of the animals was 1 year; the mass was 200-300 g. The animals were subjected to anaesthesia by the intramuscular injection 
of Zoletil 50 (Virbac, France), the dose of 0.18-0.2 ml. The hair from the skin surface was removed using the depilatory cream «Veet» (Reckitt Benckiser, France). The studies were approved by the Ethics Committee of V. I. Rasumovsky Saratov State Medical University of the Ministry of Health of Russian Federation (protocol No. 6 of 01.03 .2016 ).

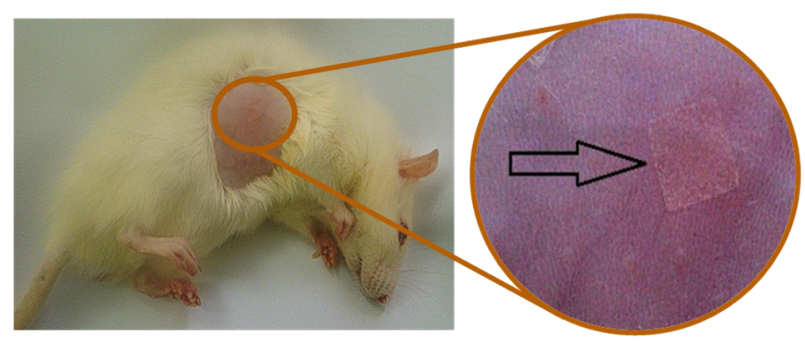

Fig. 1 Photograph of a rat and the magnified image of the skin area with the ablated upper layer fragment (pointed by an arrow).

To monitor the optical detection depth (ODD) we used the spectral optical coherence tomograph OCP930SR (Thorlabs, USA) having the following parameters: the central radiation wavelength $930 \pm 5 \mathrm{~nm}$, the radiation bandwidth at the half-maximum level 100 $\mathrm{nm}$, the longitudinal and transverse resolution in air 6.2 and $9.6 \mu \mathrm{m}$, respectively. The tomograms of the processed zone were recorded before the ablation, immediately after ablation and PEG-300 application, and then each 5 minutes during 1.5-2 hours.

Basing on the analysis of the OCT images at each moment of time, the ODD was determined, i.e., the optical distance between the skin surface and the lower bound of the useful signal, in three regions in the ablation zone. The obtained data were averaged over all animals in the group. The root-mean-square deviation was calculated.

\section{Results and Discussion}

Figure 2 (a-c) shows the typical OCT images of the intact rat skin from the group I, in 30 minutes, and 1.5 hours after the ablation. The rectangle selects the region 101 pixels wide, which nearly corresponds to $300 \mu \mathrm{m}$, over which the A-scans were averaged to measure the ODD. For the present series of images at the initial moment of time the ODD amounted to $520 \pm 10 \mu \mathrm{m}$ (Fig. $2 \mathrm{a}$ ), then in 30 minutes it became $468 \pm 10 \mu \mathrm{m}$ (Fig. 2b). The reduction of ODD is related to the slight edema in the region of ablation due to the diffusion of lymph and water from the surrounding interstitial fluid to the site of the damage, which is a natural reaction of the organism to the skin injury. However, in 1.5 hours, the increase of the ODD to $626 \pm 10 \mu \mathrm{m}$ due to the water evaporation from the ablation zone and tissue shrinkage was observed (Fig. 2c). The arrows point at the epidermis layer. One can clearly see that the ablation and consequent dehydration of tissue decreases the optical thickness of the epidermis. The mean estimated optical thickness of the epidermis amounted to $57 \pm 5 \mu \mathrm{m}, 30$ minutes later it was $42 \pm 6 \mu \mathrm{m}$, and in 1.5 hours it was $36 \pm 5 \mu \mathrm{m}$.

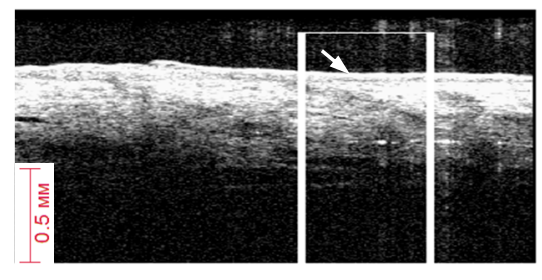

A

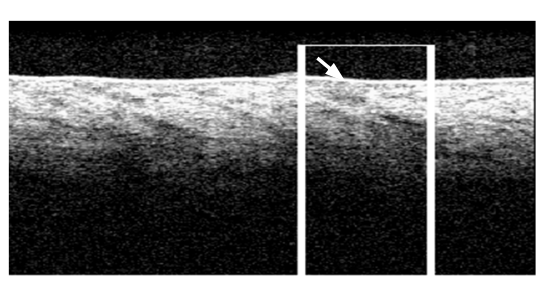

b

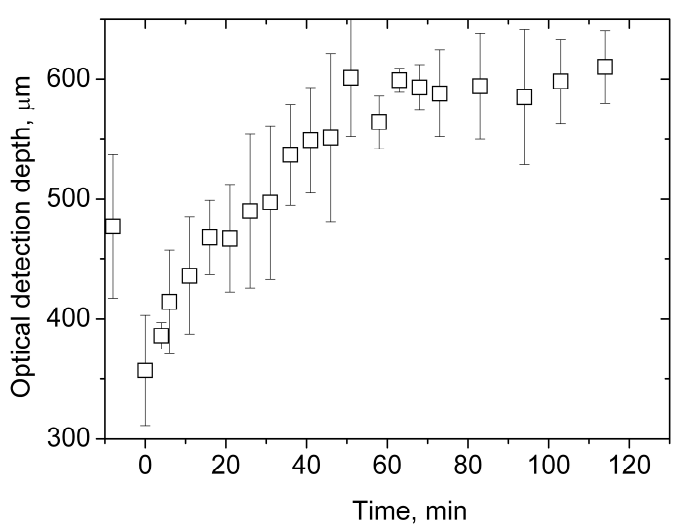

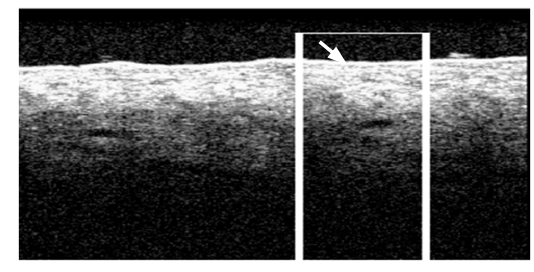

c

d

Fig. 2 OCT images of the rat skin from the first group: a - before the beginning of experiment, $\mathrm{b}-$ in 30 minutes, $\mathrm{c}-$ in 1.5 hours after the beginning of the experiment. The rectangle selects the zone of averaging A-scans of the OCT signal used to determine the optical detection depth. The arrows point at the epidermis layer. The time dependence of the averaged ODD (d). 
The kinetics of the averaged ODD is presented in Fig. 2d. The averaging was performed over 15 skin regions in five experimental animals. The vertical segments show the root-mean-square deviation. The zero abscissa corresponds to the beginning of the ODD kinetics measurement after the ablation, and the negative abscissa corresponds to the intact skin ODD. In the figure, it is clearly seen that at zero time the value of ODD is significantly smaller than before the ablation, which is a manifestation of sharp growth of light scattering by the tissue. The restoration of the ODD value to the level, corresponding to the intact skin, occurred during 30-40 min, and then the ODD increased and reached the maximal value in nearly 50-60 min after the ablation. Then up to the end of observation the ODD remained practically unchanged, which means that no more water is evaporated from the skin surface because of the formation of a dense layer in the region of damaged tissue. Sufficiently large deviation of ODD values within the group is, apparently, due to the high sensitivity of the method to insignificant breathing movements of the animal during the observation process.
Figure 3 (a-c) presents the typical OCT images of the rat skin from group II before the impact, in 30 minutes, and in 1.5 hours after applying the immersion agent. At the initial moment of time the ODD amounted to $557 \pm 10 \mu \mathrm{m}$ (Fig. 3a), and 30 minutes later it was $572 \pm 10 \mu \mathrm{m}$ (Fig. 3b). The increase of ODD occurred at the expense of the reduction of the scattering in the tissue due to the diffusion of the immersion agent into the interstitial space and the matching of the refractive indices of the interstitial fluid and collagen and elastin fibres of dermis. The ODD growth continued during the entire experiment, and in 1.5 hours the ODD reached $686 \pm 10 \mu \mathrm{m}$ (Fig. 3c). The mean optical thickness of the epidermis in this case increased from $87 \pm 4 \mu \mathrm{m}$ to $93 \pm 7$ $\mu \mathrm{m}$, since due to the penetration of propylene glycol into the interstitial space, the mean refractive index of the epidermis increased.

Figure $3 \mathrm{~d}$ presents the kinetics of the mean value of the ODD. One can clearly see that the ODD reached its maximal value in nearly $70-80 \mathrm{~min}$, and then stayed almost unchanged or even decreased. This effect can be due to the gradual reduction of the immersion agent concentration because of its diffusion to the surrounding tissue beyond the observation zone.

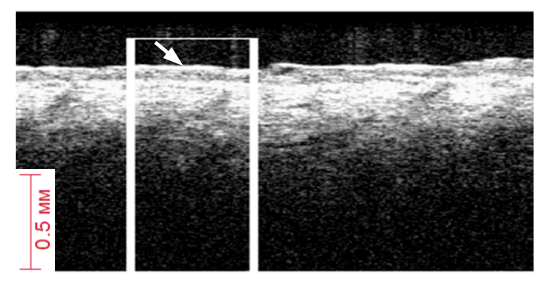

a

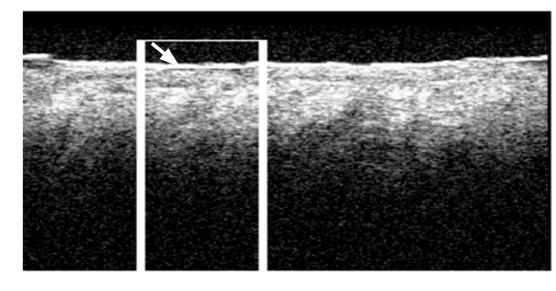

b

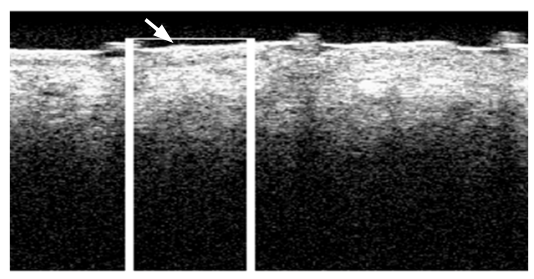

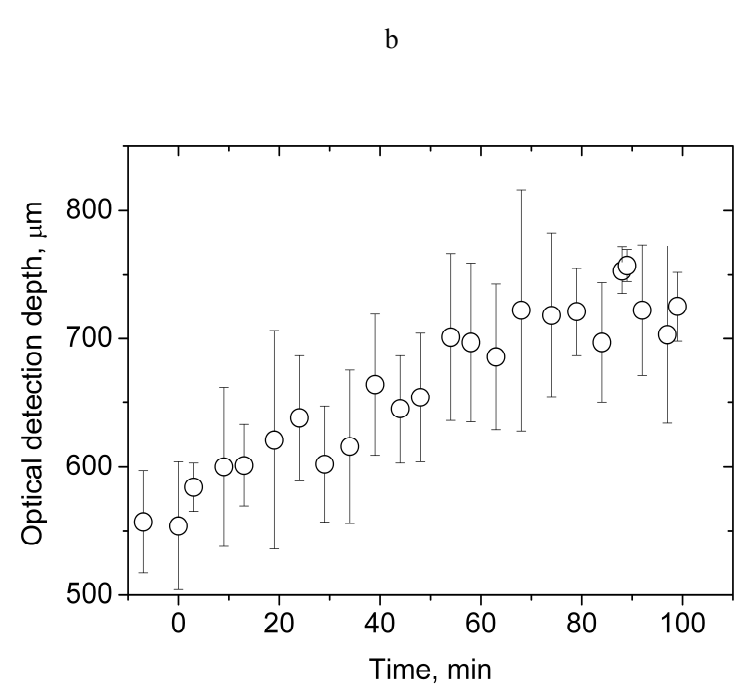

d

Fig. 3 OCT image of the rat skin from group II: $\mathrm{a}$ - before the beginning of experiment, $\mathrm{b}-$ in 30 minutes, $\mathrm{c}-$ in 1.5 hours after the beginning of experiment. The rectangle selects the zone of A-scans averaging and measuring the optical detection depth. The arrows point at the epidermis layer. Time dependence of the averaged ODD (d).

Figure 4 (a-c) shows the typical OCT images of the intact rat skin from group III before the impact, in 30 minutes, and in 1.5 hours after the ablation and application of immersion agent. At the initial moment the ODD amounted to $512 \pm 10 \mu \mathrm{m}$ (Fig. $4 \mathrm{a}$ ), and in 30 minutes it was $635 \pm 10 \mu \mathrm{m}$ (Fig. 4b). The mechanism of optical clearing incorporated two competing processes. On the one hand, the scattering increased due to the influx of water and lymph from the surrounding interstitial fluid to the site of injury. On the other hand, 
the scattering decreased due to the impact of the immersion agent; the growth of its concentration in the interstitial fluid matched the refractive indices of the interstitial fluid and the collagen fibres of the dermis. As a result, in 1.5 hours the ODD increased to $701 \pm 10 \mu \mathrm{m}$ (Fig. 4c). The estimated optical thickness, first, decreased from $72 \pm 6 \mu \mathrm{m}$ to $63 \pm 5 \mu \mathrm{m}$ because of the partial removal of the epidermal layer. Then it gradually grew to achieve $78 \pm 6 \mu \mathrm{m}$ (in 1.5 hours) because of the increase of the mean refractive index of the tissue due to the penetration of polyethylene glycol, like in the group II.

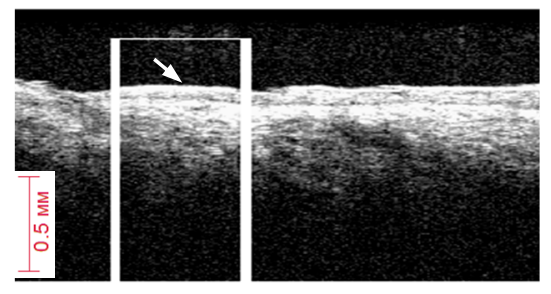

a

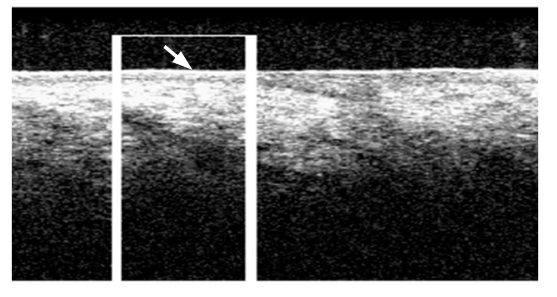

b

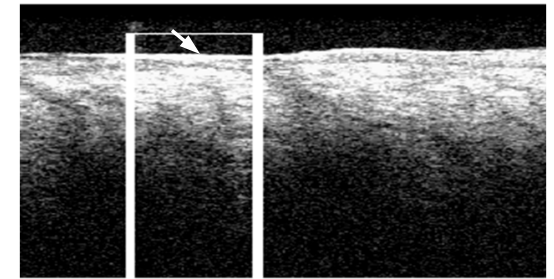

$\mathrm{c}$

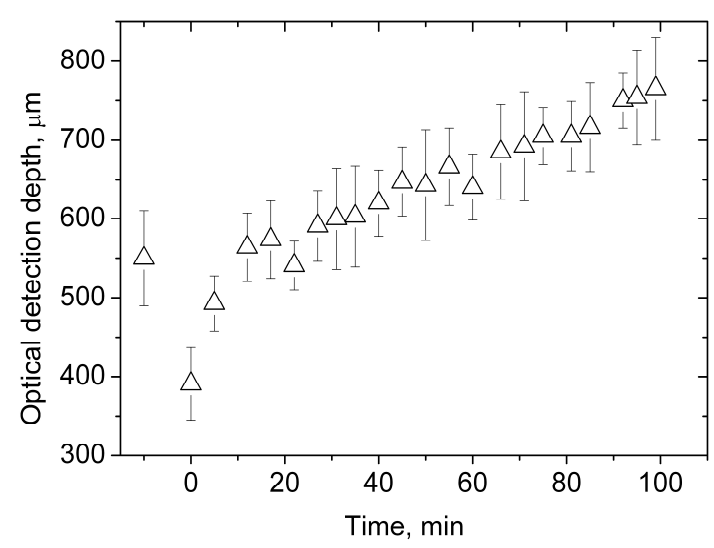

d

Fig. 4 OCT image of the rat skin from group III: a - before the beginning of the experiment, $\mathrm{b}-$ in 30 minutes, $\mathrm{c}-$ in 1.5 hours after the beginning of the experiment. The rectangle selects the zone of A-scans averaging and determination of the optical detection depth. The arrows point at the epidermis layer. Time dependence of averaged ODD (d).

The kinetics of the group-averaged ODD presented in Fig. 4d shows that, like in the first group, the growth of the ODD began from the lower values, as compared to the second group, due to the appearance of skin edema because of its damage. However, the restoration of the ODD to the initial values occurred significantly faster (during nearly 15-20 minutes) than in the first group. In the present case, no water evaporated from the skin surface, since the surface was covered with a layer of PEG-300. The ODD growth may be attributed only to the reduction of skin scattering because of immersion.

Figure 5 presents the time dependence of ODD for three groups of experimental animals in the process of observation, normalised to the skin ODD before the ablation and/or the application of PEG-300 (the negative abscissa values). The moment of time $t=0$ corresponds to the beginning of the recording of the optical clearing kinetics. The deviation of values with respect to the normalised mean value is not shown here for clarity, since the curves are rather close to each other.

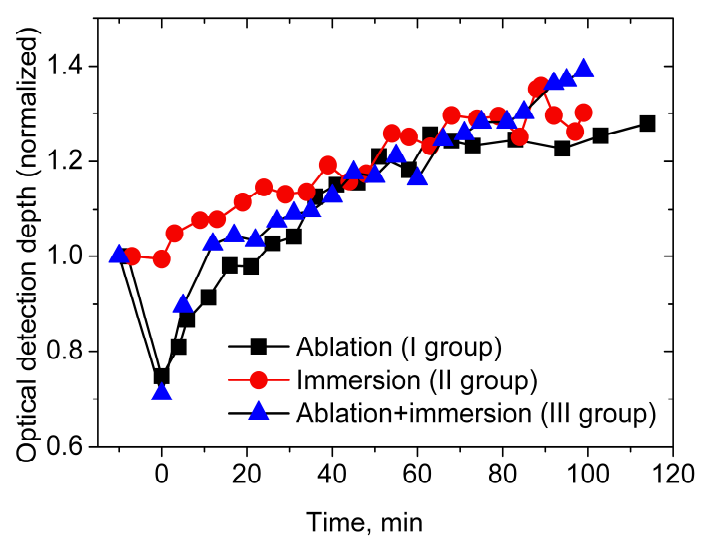

Fig. 5 Time dependence of ODD normalised to that of the intact skin. The symbols correspond to the experimental data.

Basing on the presented results one can estimate the reduction of the detection depth due to the ablation as $25-30 \%$, which to our opinion is related to the increased light scattering in the skin at the ablation zone due to the appearance of edema. From the comparison of curves, corresponding to groups I and III it is seen that the 
application of PEG-300 to the skin surface leads to insignificant lowering of the ODD, apparently, related to the probe radiation shielding by the layer of immersion agent.

From the comparison of kinetic curves, it also follows that the rate of optical clearing in groups I and III is higher than in group I, since the slope of the first and the third curve in the region from 0 to approximately $60 \mathrm{~min}$ is greater than that of the curve corresponding to the second group. One can explain this fact by the protective properties of the epidermis that inhibit the penetration of PEG-300 into the skin. It is also clearly seen that the slope of the third curve is maximal. Besides that, the comparison of the ODD variation kinetics shows that nearly in an hour after the ablation in the first and the second group the ODD becomes stabilised and remains practically unchanged, while in the animals of group III the ODD continues to increase, which demonstrates the dominant influence of PEG-300 on the ODD.

The increase of skin permeability both for water, leaving the skin in the process of evaporation, and for the preparations applied to its surface, due to the reduction of the epidermis thickness or to the epidermis removal by ablation, was reported in Refs. [15-18]. The appearance of skin edema in the zone of fractional microablation was detected using the backscattering spectra [19]. It was noticed that the residual edema stayed for 24 hours after the ablation. However, the kinetics of measurement of the OCT optical detection depth under the simultaneous formation of post-ablation edema and tissue immersion is studied in this paper for the first time. We have shown that after the ablation of the epidermis using a wide beam, the edema arising as a protective reaction of the organism to the injury, significantly reduces the depth of light penetration into the skin. To our opinion, this is due to the increased light scattering by dermis, mainly due to the reduction of the refractive index of interstitial fluid, i.e., the additional mismatch of refractive indices of dermis components caused by the influx of the interstitial fluid and lymph to the site of injury.

\section{Conclusion}

The results of the study of optical clearing of rat skin in vivo based on the kinetics of the OCT optical detection depth have shown that the epidermis ablation does not increase the ODD during 100-110 minutes. Moreover, at the initial stage (30-40 minutes) it even leads to the ODD reduction by $25-30 \%$. Thus, although the permeability of epidermis for the immersion agent increases, the light scattering by dermis increases because of the edema, which inhibits the optical clearing of skin. This effect does not allow one to use the wide-beam ablation to increase the rate of optical clearing of skin. The reduction of edema degree and the increase of the skin optical clearing rate could be possible by using the fractional microablation that produces a matrix of microscopic channels in the skin [10]. However, this hypothesis requires experimental testing.

The authors express their gratitude to L.E. Dolotov for the help in performing experiments and to A.B. Bucharskaya for providing them with laboratory animals. The study was carried out within the project supported by the President of Russia, Grant NSh7898.2016.2. 